Open Access

\title{
Identification of altered protein abundances in cholesteatoma matrix via mass spectrometry-based proteomic analysis
}

\author{
Derrick R. Randall, Phillip S. Park and Justin K. Chau*
}

\begin{abstract}
Background: Cholesteatoma are cyst-like structures lined with a matrix of differentiated squamous epithelium overlying connective tissue. Although epithelium normally exhibits self-limited growth, cholesteatoma matrix erodes mucosa and bone suggesting changes in matrix protein constituents that permit destructive behaviour. Differential proteomic studies can measure and compare the cholesteatoma proteome to normal tissues, revealing protein alterations that may propagate the destructive process.

Methods: Human cholesteatoma matrix, cholesteatoma-involved ossicles, and normal middle ear mucosa, post-auricular skin, and non-involved ossicles were harvested. These tissues were subjected to multiplex peptide labeling followed by liquid chromatography and tandem mass spectrometry analysis. Relative protein abundances were compared and evaluated for ontologic function and putative involvement in cholesteatoma.
\end{abstract}

Results: Our methodology detected 10764 peptides constituting 1662 unique proteins at $95 \%$ confidence or greater. Twenty-nine candidate proteins were identified in soft tissue analysis, with 29 additional proteins showing altered abundances in bone samples. Ontologic functions and known relevance to cholesteatoma are discussed, with several candidates highlighted for their roles in epithelial integrity, evasion of apoptosis, and immunologic function.

Conclusion: This study produced an extensive cholesteatoma proteome and identified 58 proteins with altered abundances contributing to disease pathopathysiology. As well, potential biomarkers of residual disease were highlighted. Further investigation into these proteins may provide useful options for novel therapeutics or monitoring disease status.

\section{Background}

Cholesteatoma is a benign epidermal inclusion cyst that develops within the temporal bone and exhibits locally destructive behavior. Without treatment, cholesteatomas can progressively expand and destroy middle ear and temporal bone structures. This process can lead to secondary infections, which can result in complications such as tympanic membrane perforation, chronic otorrhea, hearing loss, vestibular dysfunction, facial nerve

\footnotetext{
* Correspondence: justin.chau@gmail.com
This article was presented at the Canadian Society of Otolaryngology - Head

* Correspondence: justin.chau@gmail.com \& Neck Surgery Poliquin Competition, Winnipeg, MB, June 8, 2015 Section of Otolaryngology - Head \& Neck Surgery, Department of Surgery,
University of Calgary, Calgary, Foothills Medical Centre, 1403 - 29 Street NW Section of Otolaryngology - Head \& Neck Surgery, Department of Surgery,
University of Calgary, Calgary, Foothills Medical Centre, 1403 - 29 Street NW, Calgary, AB T2N 2T9, Canada
}

paresis, and intracranial extension [1]. Regardless of surgical technique, cholesteatoma have propensity to recur [2], requiring exteriorization of the middle ear through canal wall down procedures or "second look" tympanomastoidectomy for surveillance of disease in intact canal wall procedures.

Increased understanding of molecular changes in bone through orthopedic and otitis media literature led to theories of cholesteatoma-related bone destruction through cellular resorption, mechanical compression, and second mediator effects [2-7]. Bone erosion involved in the progression of disease may be intrinsic to the cholesteatoma-increased matrix growth factor and cytokine expression, pressure effect of outward growth, and host granulation enzymes. Extrinsic factors include 
bacterial superinfection, altered osteoclast activity in response to invasion, and changes in bone architecture and cell population $[3,7,8]$. Numerous targeted molecular and genome wide studies on cholesteatoma specimens support some of these hypotheses but fail to appreciate the complex interplay between multiple changes at the cellular level.

Differential proteomic analysis evaluates the active protein constellation between normal and pathologic states [9]. Initiated as two-dimensional gel electrophoresis (2DGE) with densitometry or visual evaluation, modern proteomic analysis evolved to incorporate massspectrometry in protein identification from individual 2DGE studies to current chromatographic separation and tandem mass spectrometry techniques capable of analyzing multiple tissue conditions simultaneously. Modern mass spectrometry-based techniques have improved the ability to recognize novel, replicable protein derangements in various diseases and tissues by comparing disease to normal states [10]. These changes can be detected among proteins expressed at very low levels and several orders of magnitude below that of the most abundant proteins.

Previous proteomic approaches to cholesteatoma identified several proteins with altered presence in comparison to post-auricular skin through 2DGE [11]. In this study, we employed a multiplex differential mass spectrometry-based approach termed isotopetagged relative abundance quantification (iTRAQ) to characterize simultaneously the cholesteatoma matrix proteome in reference to native middle ear mucosa and post-auricular skin as well as to evaluate the bone proteome of ossicles involved by cholesteatoma compared to normal ossicles. With this design we were able to detect agents underlying the pathophysiologic process and destructive behavior demonstrated by cholesteatoma as well as identify and quantify potential biomarkers of disease.

\section{Methods}

\section{Sample collection}

This study received ethical approval from the University of Calgary Conjoint Health Research Ethics Board (REB14-0883). Patient tissue samples were obtained during primary tympanomastoidectomy from patients with acquired cholesteatoma, whereby cholesteatoma matrix was excised and cleaned of keratin debris; middle ear mucosa was collected from uninvolved regions of the mastoid cavity; post-auricular skin was taken as a $1 \mathrm{~mm}$ wide strip of skin along the margin of the skin incision with subcutaneous tissue removed by sharp dissection under an operating microscope. Cholesteatoma-involved ossicles were harvested during tympanomastoidectomy from patients with evidence of ossicular destruction. Control ossicles were obtained from patients undergoing labyrinthectomy for vestibular schwannoma or ossicular reconstruction for traumatic ossicular discontinuity. Tissue samples were immediately placed on ice then stored at $-80{ }^{\circ} \mathrm{C}$ until sample processing.

\section{Protein extraction}

Samples were thawed on ice then washed three times in $4{ }^{\circ} \mathrm{C}$ phosphate-buffered saline containing complete protease inhibitor (Roche, Mississauga, ON). Sample processing was performed by the following methods for either soft tissue or bone, in an identical fashion for each tissue sample, with all buffers containing c0mplete protease inhibitor (Roche, Mississauga, ON).

Soft tissue samples were pooled as mucosa, matrix, or skin samples. Each pool was incubated at $4{ }^{\circ} \mathrm{C}$ for $16 \mathrm{~h}$ in modified RIPA buffer with protease inhibitor. Samples were then sonicated on ice for $7 \mathrm{~s}$ at $20 \%$ power for $5 \mathrm{cy}$ cles with $30 \mathrm{~s}$ cooling periods. The resultant lysates were centrifuged for $60 \mathrm{~min}$ at $12000 \mathrm{~g}$ at $4{ }^{\circ} \mathrm{C}$ in an Allegra $\mathrm{X}-15 \mathrm{R}$ centrifuge (Beckman-Coulter, Mississauga, ON) and the supernatant preserved. Equivalent total protein concentrations from each pool were precipitated in $100 \%$ chilled ethanol (Sigma, Oakville, ON) by incubating $24 \mathrm{~h}$ at $-20{ }^{\circ} \mathrm{C}$ followed by $20 \mathrm{~min}$ centrifugation at $2500 \mathrm{~g}\left(4{ }^{\circ} \mathrm{C}\right)$. The pellet was preserved, washed once with $80 \%$ ethanol $(\mathrm{v} / \mathrm{v})$, centrifuged at $2500 \mathrm{~g}\left(4{ }^{\circ} \mathrm{C}\right)$, decanted, and air dried for $5 \mathrm{~min}$. The precipitated samples were stored at $-80{ }^{\circ} \mathrm{C}$ until iTRAQ analysis.

Individual bone samples were incubated at room temperature in $0.06 \mathrm{M}$ hydrocholoric acid $(\mathrm{HCl})$ for $16 \mathrm{~h}$. The diluent (D1) was removed and the bone samples incubated in $1.2 \mathrm{M} \mathrm{HCl}$ at $4{ }^{\circ} \mathrm{C}$ for $16 \mathrm{~h}$ to decalcify the bone. After decalcification, the diluent (D2) was removed, and bone samples were pooled into two groups (control ossicles or cholesteatoma-involved ossicles) then washed with extraction buffer 1 (E1: $6 \mathrm{M}$ guanidine- $\mathrm{HCl}$ and $100 \mathrm{mM}$ Tris- $\mathrm{HCl}$ with protease inhibitor). Buffer E1 was added to the pooled samples, which were sonicated on ice for $7 \mathrm{~s}$ at $20 \%$ power for 5 cycles with $30 \mathrm{~s}$ cooling periods. Samples were incubated for $72 \mathrm{~h}$ at $4{ }^{\circ} \mathrm{C}$ then centrifuged for $20 \mathrm{~min}$ at $12000 \mathrm{~g}\left(4^{\circ} \mathrm{C}\right)$. Supernatant $\mathrm{E} 1$ was removed and stored at $-80^{\circ} \mathrm{C}$. Samples were washed with extraction buffer 1.5 (E1.5: $6 \mathrm{M}$ guanidine- $\mathrm{HCl}, 100 \mathrm{mM}$ HEPES, $0.5 \mathrm{M} \mathrm{Na}_{4}$ EDTA, protease inhibitor), then buffer E1.5 was added to the pellet, agitated, and incubated $72 \mathrm{~h}$ at $4{ }^{\circ} \mathrm{C}$. Samples were centrifuged for $20 \mathrm{~min}$ at 12 $000 \mathrm{~g}$ at $4{ }^{\circ} \mathrm{C}$, and supernatant $\mathrm{E} 1.5$ removed and stored at $-80{ }^{\circ} \mathrm{C}$. Samples were washed with extraction buffer 2 (E2: $6 \mathrm{M} \mathrm{HCl}, 6 \mathrm{M}$ guanidine- $\mathrm{HCl}, 100 \mathrm{mM} 100 \mathrm{mM}$ Tris, protease inhibitor), then buffer E2 was added to the pellet, fragmented, and incubated $20 \mathrm{~h}$ at $4{ }^{\circ} \mathrm{C}$. Samples D2, E1, E1.5, and E2 were combined in their respective pooled samples and precipitated by the same 
method as the soft tissue samples and stored at $-80{ }^{\circ} \mathrm{C}$ until iTRAQ analysis.

\section{ITRAQ reagent preparation}

Two LC-MS/MS tracts were defined and run on separate days in order to minimize the impact of high abundance proteins from each tissue type limiting the ability to detect low abundance proteins: one for soft tissue and one for bone samples. Soft tissue and bone lysates were labelled with iTRAQ reagent according to the University of Victoria Protein Centre protocols. $100 \mu \mathrm{g}$ of total protein from each sample underwent acetone precipitation followed by resuspension in iTRAQ buffer and trypsin digestion. Each protein lysate was labeled with a distinct isotopic iTRAQ reagent: control ossicles $(113 \mathrm{Da})$, cholesteatoma-involved ossicles (115 Da); cholesteatoma matrix 1 (113 Da), cholesteatoma matrix 2 (114 Da), mucosa (115 Da), post-auricular skin (116 Da). These samples were combined and subjected to alkaline $(\mathrm{pH} 10)$ reversed phase high performance liquid chromatography on an XBridge C18 BEH300 $250 \mathrm{~mm} \mathrm{X}$ $4.6 \mathrm{~mm}, 5 \mu \mathrm{m}$, 300A HPLC column (Waters, MA, USA), with fractions collected every minute for $96 \mathrm{~min}$.

\section{LC-MS/MS analysis}

Fractions were separated by on-line reversed phase liquid chromatography using a Thermo Scientific EASYnanoLC II system with a reversed-phase pre-column Magic C-18AQ (100 $\mu \mathrm{m}$ I.D., $2 \mathrm{~cm}$ length, and an inhouse prepared reversed-phase nano-analytical column packed with Magic C-18AQ (75 $\mu \mathrm{m}$ I.D., $15 \mathrm{~cm}$ length, $5 \mu \mathrm{m}, 100 \AA$ Aं Michrom BioResources Inc, Auburn, CA). The chromatography system was coupled on-line to an LTQ Orbitrap Velos Pro mass spectrometer equipped with a Nanospray Flex source (Thermo Fisher Scientific, Bremen, Germany) and run over a 120 min gradient from $95 \%$ solvent A (2 \% Acetonitrile, $0.1 \%$ Formic acid):5 \% solvent B (90 \% Acetonitrile, $0.1 \%$ Formic acid) to $100 \%$ solvent B. Mass spectrometry data were acquired with a time of flight survey scan of mass range 400-1800 amu where the most abundant ions exceeding 5000 counts and charge state $2-4$ selected for fragmentation.

\section{Data acquisition and analysis}

The resulting data were analyzed by Proteome Discoverer 1.4 software suite (Thermo Scientific) using stringency criteria: $\mathrm{s} / \mathrm{n}$ cut-off: 1.5 ; total intensity threshold: 0 ; minimum peak count: 1; precursor mass: 350-5000 Da. The peak lists were submitted to an in-house database search using Mascot 2.4 (Matrix Science), and were searched against the Uniprot-Swissprot database (May 30, 2015 update; 540,261 sequences; 191,876,607 residues) assuming 2 or less missed trypsin cut sites as well as fixed methylthio and variable oxidation and deamidation modifications.
Target false discovery rate was set a 0.01 . All identified proteins were assessed for gene ontology functions with an additional function set for structural components and those proteins with bone-related functions. Candidate proteins were selected if their relative abundances were greater than 1.5-fold ( $\log _{2}$ scale) increased or decreased in cholesteatoma matrix compared to either middle ear mucosa or post-auricular skin for soft tissue analysis, or if proteins in cholesteatoma-involved bone were greater than 1.5-fold ( $\log _{2}$ scale) increased or decreased relative to control ossicles and found to have ontologic functions corresponding to cellular metabolism, bone structure, bone metabolism, or epithelial content.

\section{Results}

The soft tissue arm of our study included representative samples from 12 cholesteatoma matrices, eight nondiseased middle ear mucosa fragments, and nine postauricular skin samples. Healthy mucosa not involved with cholesteatoma could not be identified in four patients and was not collected; three post-auricular skin samples were suboptimal samples and not included. iTRAQ analysis of the included samples detected 10720 spectra representing 8372 unique peptides. Setting stringency at $95 \%$ probability of protein recognition and at least two peptides per protein identified 1490 proteins from 8315 of these peptides, corresponding to an average of 5.58 peptides per protein. The ontologic functions of these proteins are displayed in Fig. 1a. Cellular metabolism, signal transduction, and DNA replication/transcription/repair functions comprise more than $50 \%$ of the identified proteins.

With respect to the bone lysate analysis, which included eight cholesteatoma-involved ossicles and eight control ossicles, 3159 spectra and 2392 unique peptides produced 480 proteins with at least two peptides and $95 \%$ confidence of identification (4.96 peptides per protein). After subtracting protein clusters representing homologous protein family members, 428 were recognized with greater than $95 \%$ confidence and further scrutinized. Gene ontology functions for the identified proteins are shown in Fig. 1b. The most common functions identified were metabolism, signal transduction, and bone structure, which accounted for $50 \%$ of all proteins; $10 \%$ of proteins played a role in bone structure and another $7 \%$ in bone metabolism. In comparison to the soft tissue analysis there were 256 common proteins, indicating our study identified 1662 proteins from cholesteatoma and involved middle ear structures.

Figures 2 and 3 display the proteins identified in the soft tissue analysis with relative abundances of proteins in mucosa and skin, respectively, compared to cholesteatoma matrix. Proteins exceeding the relative abundance threshold are highlighted in red and represent potential 


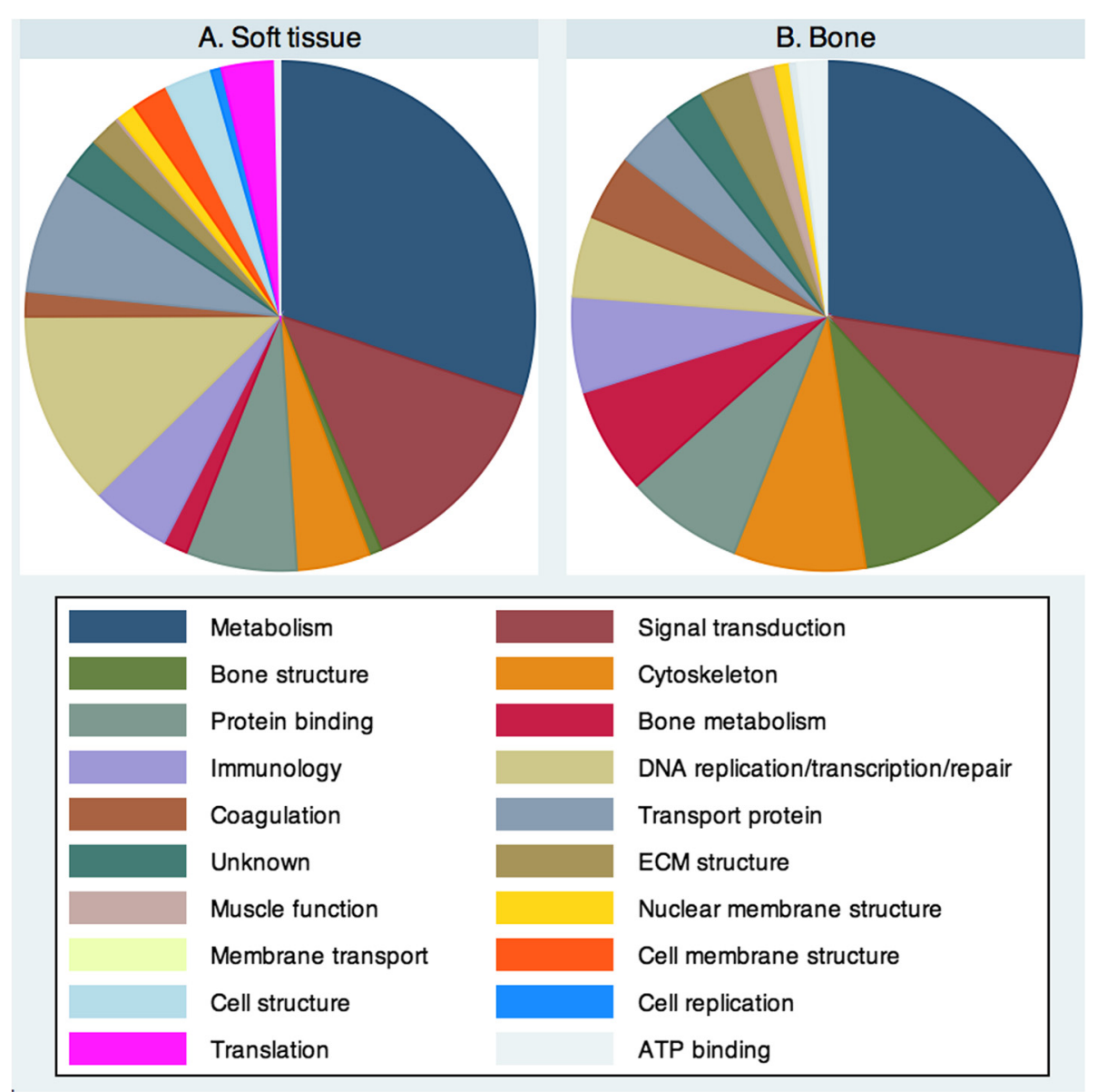

Fig. 1 Ontologic functions of proteins identified in (a). Soft tissue analysis and (b). Bone analysis. General metabolic function and signal transduction properties represent the most common ontologic functions in both tissue sets, but wide variation exists among the remaining categories

candidates in cholesteatoma pathophysiology or biomarkers of disease. These 29 proteins are listed in Table 1 with their relative abundances in cholesteatoma matrix in reference to both uninvolved mucosa and post-auricular skin. Positive numbers indicate increased abundance in cholesteatoma while negative values indicate a higher relative abundance in normal tissue. Similarly, Fig. 4 shows all proteins identified in the bone analysis with potential candidates based on relative abundance alterations marked; Table 2 contains the 33 proteins marked in Fig. 4 sorted by relative abundance in cholesteatoma-involved ossicles to uninvolved ossicles. Four proteins were present in altered abundances in both the soft tissue and bone tissue analyses: creatine kinase B-type (CKB), tenascin$\mathrm{X}$ (TNXB), serum amyloid P-component (APCS), and keratin type 2 cytoskeletal 8 (KRT8).

\section{Discussion}

Our study used a mass spectrometry-based proteomic approach to evaluate the relative abundance changes between proteins found within middle ear mucosa and postauricular skin relative to cholesteatoma matrix. A second arm of the study investigated the protein changes occurring in bone involved with cholesteatoma relative to healthy ossicles. We identified a number of potential candidates in the molecular changes among the functional components of the cholesteatoma matrix leading to uncontrolled growth and bony destruction.

Recognizing proteins with either increased or decreased abundance in both skin and mucosa reflect deviations from normal, particularly when they are functional rather than primarily structural in nature. Proteins fitting this description include members of the S100 family (A7, A8, and A9) and SERPINB3. S100 proteins are elevated in hyperproliferative skin disorders with primary roles in cell cycle regulation and cell differentiation [12, 13]. S100A7 also functions in an antibacterial capacity through the Toll-like receptor pathway in response to the bacterial protein flagellin [14], which is found in P. aeruginosa and E. coli, among other bacteria. SERPINB3 is a serine 


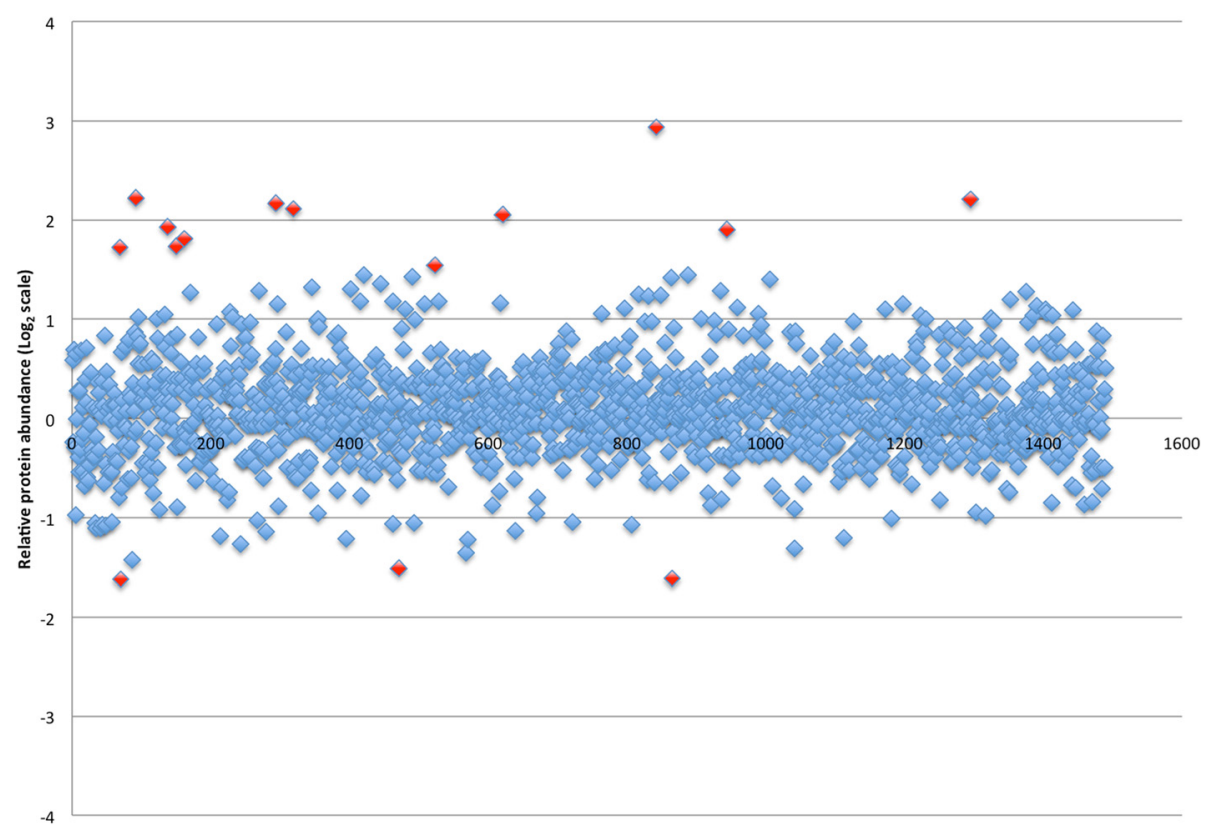

Fig. 2 Logarithm (base 2) plot of relative abundances of all proteins identified in cholesteatoma matrix in reference to control mucosa. Positive values indicate greater protein content in cholesteatoma. The 15 proteins exceeding threshold abundances are indicated in red

protease inhibitor associated with cellular atypia, evasion of apoptosis, and cholesteatoma proliferation [15-17]. Our identification of SERPINB3 and the three S100 proteins in cholesteatoma matrix agrees with existing literature noting increased levels in cholesteatoma relative to post-auricular skin $[11,18]$, but it is a potentially novel finding to find increased abundance relative to middle ear mucosa. Though adult cholesteatoma is a disease of keratinizing epithelium that likely originates from the lateral surface of the tympanic membrane or external auditory canal, its destructive properties occur primarily within the middle ear; as such, finding difference between the

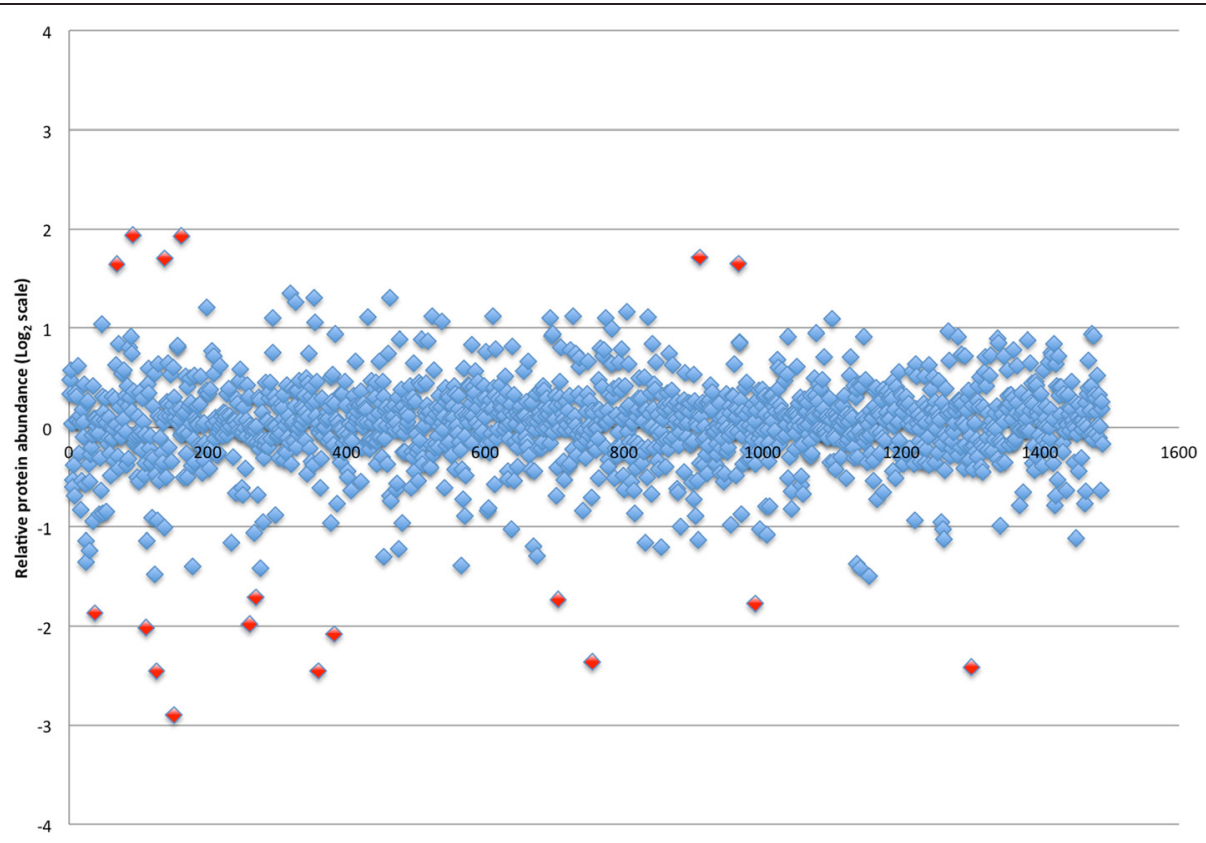

Fig. 3 Logarithm (base 2) plot of relative abundances of all proteins identified in cholesteatoma matrix in reference to post-auricular skin. Positive values indicate greater protein content in cholesteatoma. The 18 proteins exceeding threshold abundances are indicated in red 
Table 1 Altered soft tissue protein abundances in cholesteatoma matrix relative to normal mucosa and post-auricular skin. Positive values indicate a greater abundance of a protein in cholesteatoma than the reference tissue. Italicized relative abundances indicate values below the detection threshold for one tissue but above detection threshold in the other. Study ID numbers correlate to Figs. 2 and 3

\begin{tabular}{|c|c|c|c|c|c|}
\hline \multirow[t]{2}{*}{ Protein name (study ID number) } & \multirow{2}{*}{$\begin{array}{l}\text { NCBI gene } \\
\text { ID }\end{array}$} & \multirow{2}{*}{$\begin{array}{l}\text { Number of unique } \\
\text { peptides }\end{array}$} & \multirow{2}{*}{$\begin{array}{l}\text { Percent protein } \\
\text { coverage (\%) }\end{array}$} & \multicolumn{2}{|c|}{ Relative protein abundance } \\
\hline & & & & Mucosa & Skin \\
\hline Bleomycin hydrolase (842) & BLMH & 4 & 13.8 & 7.66 & 1.14 \\
\hline Protein S100-A9 (92) & S100A9 & 5 & 55.3 & 4.66 & 3.83 \\
\hline Acyl-coenzyme A thioesterase 1 (1295) & ACOT1 & 2 & 5.7 & 4.62 & -1.26 \\
\hline Filaggrin (294) & FLG & 10 & 3.7 & 4.50 & 2.14 \\
\hline FA binding protein, epidermal (319) & FABP5 & 7 & 65.2 & 4.32 & 2.55 \\
\hline Filaggrin-2 (621) & FLG2 & 3 & 1.0 & 4.15 & 1.35 \\
\hline Protein S100-A7 (138) & S100A7 & 7 & 54.5 & 3.81 & 3.25 \\
\hline Creatine kinase B-type (944) & CKB & 2 & 7.9 & 3.74 & 1.47 \\
\hline Protein S100-A8 (162) & S100A8 & 5 & 64.5 & 3.50 & 3.80 \\
\hline Thymidine phosphorylase (150) & TYMP & 10 & 35.1 & 3.32 & 1.52 \\
\hline Serine protease inhibitor B3 (69) & SERPINB3 & 22 & 50.3 & 3.30 & 3.12 \\
\hline Myelin protein P0 (523) & MPZ & 6 & 25.0 & 2.92 & 1.06 \\
\hline Desmocollin-3 (989) & DSC3 & 3 & 4.2 & 2.07 & -3.42 \\
\hline Ferritin light chain (965) & FTL & 3 & 19.4 & 1.42 & 3.13 \\
\hline Fatty acid desaturase 2 (1301) & FADS2 & 2 & 3.6 & 1.32 & -5.34 \\
\hline Hydroxymethylglutaryl-CoA synthase, cytoplasmic (705) & HMGCS1 & 2 & 6.0 & 1.30 & -3.32 \\
\hline Fatty acid synthase (37) & FASN & 38 & 23.7 & 1.29 & -3.65 \\
\hline Collagen alpha-1(III) chain (754) & COL3A1 & 3 & 2.7 & 1.26 & -5.15 \\
\hline Collagen alpha-6(VI) chain (260) & COL6A6 & 10 & 7.0 & 1.18 & -3.95 \\
\hline Integrin alpha-M (909) & ITGAM & 4 & 3.9 & 1.16 & 3.28 \\
\hline Keratin, type II cytoskeletal 79 (359) & KRT79 & 15 & 27.7 & -1.03 & -5.48 \\
\hline Tenascin-X (111) & TNXB & 23 & 8.2 & -1.18 & -4.05 \\
\hline Serum amyloid P-component (269) & APCS & 5 & 23.3 & -1.23 & -3.27 \\
\hline Tryptase alpha/beta-1 (382) & TPSAB1 & 4 & 14.5 & -1.65 & -4.23 \\
\hline Collagen alpha-2(I) chain (151) & COL1A2 & 7 & 54.5 & -1.86 & -7.48 \\
\hline Mimecan/osteoglycin (126) & OGN & 7 & 24.8 & -1.89 & -5.49 \\
\hline Keratin type 2 cytoskeletal 8 (471) & KRT8 & 13 & 21.7 & -2.85 & -1.55 \\
\hline BPI fold containing family member B (865) & BPIFB1 & 3 & 8.5 & -3.06 & 1.68 \\
\hline Fibrillin-1 (70) & FBN1 & 27 & 11.3 & -3.08 & -1.23 \\
\hline
\end{tabular}

cholesteatoma matrix that allows it to overgrow mucosa and destroy neighbouring tissue may be more appreciable by analyzing the mucosa of presumably unaffected middle ear tissue.

A biomarker that is able to distinguish normal mucosa from cholesteatoma could prove useful for intra-operative tissue labeling or frozen section pathologic analysis, aiding the surgeon in removing all diseased tissue. Therefore another objective of this study was to recognize proteins that may function as indicators of residual or recurrent disease. As expected, the landscape of the cholesteatoma matrix proteome resembles that of normal skin, with multiple low-level abundance changes that likely reflect a complex interplay between various proteins and gene expression levels. The ideal candidate protein profile for this would have increased relative abundance in cholesteatoma compared to mucosa. Of the novel protein alterations we identified, some of the most intriguing changes involve BLMH, TYMP, FLBP5, and FLG/FLG2. BLMH represents a good candidate since it is located in superficial epidermis and maintains epithelial integrity [19]. Increased BLMH levels in cholesteatoma relative to mucosa, but unchanged in skin, is logical given that it is found primarily in the superficial and corneal layers of epidermis which are largely deficient in the non-keratinizing middle ear epithelium. This makes it an excellent potential biomarker 


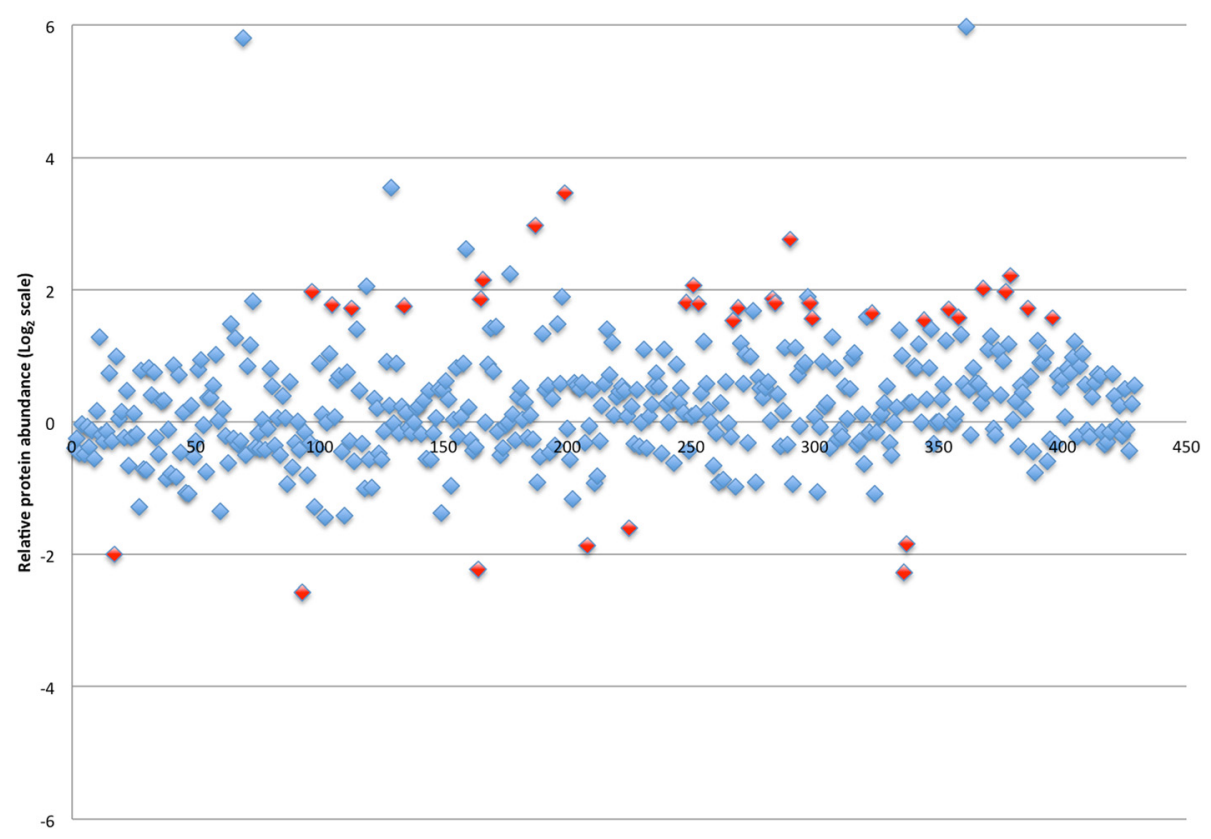

Fig. 4 Logarithm (base 2) plot of relative abundances of all proteins identified in cholesteatoma-involved ossicles in reference to control ossicles. Positive values indicate greater protein content in cholesteatoma-involved bone. The 34 proteins exceeding threshold abundances and meeting inclusion criteria are highlighted in red

since presence in the middle ear following cholesteatoma excision may represent residual disease. Likewise, FLG is long recognized as elevated in cholesteatoma by histopathologic studies relative to post-auricular skin $[20,21]$, and we note considerably greater degree of elevation in cholesteatoma compared to mucosa with similar change noted in FLG2, which is co-expressed with FLG during keratinocyte differentiation [22]. Previous data suggests an interaction between FLG and involucrin (IVL), a protein that creates a protective envelope around corneocytes, and increased IVL in cholesteatoma [21, 23]; we identified increased IVL in cholesteatoma matrix, but not at levels meeting detection threshold (1.97-fold increase).

TYMP abundance was elevated in cholesteatoma 3.25fold in our study compared to mucosa, though less so compared to post-auricular skin. TYMP is an angiogenesis factor involved in avoiding hypoxia-induced apoptosis, though most research focuses on malignant disease [24]. The role of hypoxia in cholesteatoma is poorly understood and thought to stimulate matrix metalloprotease release and subsequent perimatrix degeneration [25]. Elevated TYMP may contribute to the independent growth capability of cholesteatoma particularly when oxygen requirements exceed that available from direct diffusion in aerated portions of the middle ear. In addition to oxygen supply, energy demand is another hurdle cholesteatoma must overcome to continue growth and local destructive potential. Fatty acid metabolism is gaining attention in cholesteatoma pathophysiology as an available energy source to propagate self-sufficient growth [25]. We found epidermal fatty acid binding protein (FABP5) is highly overabundant in cholesteatoma compared to normal mucosa (4.3-fold), with a lesser increase compared to skin. Initially identified in psoriasis, FABP5 is unique to keratinocytes, is a key factor in keratinocyte differentiation, and interacts with other proteins found at altered abundances in our study [26]. Of note, increased FABP5 stimulates increased IVL and is stabilized by S100A7 [27, 28]. FABP5 also appears to have a role in inflammatory change and tissue injury involving keratinocytes [29].

Other notable observations are the reduction of several structural proteins in cholesteatoma compared to postauricular skin. These proteins provide insight into the molecular changes occurring that cause the well-recognized fragility of matrix intra-operatively. Desmocollin-3 (DSC3) likely contributes to this friability, as loss of function is found in skin fragility disorders [30], which matches the abundance profile we observed with decreased levels (3.42-fold) in cholesteatoma compared to skin, but above that seen in mucosa. Intercellular tight junction loss in cholesteatoma compared to normal skin has been shown by electrical impedence studies, agreeing with the impaired intercellular connections we found [31]. As shown in Table 1, a large proportion of the proteins reduced in cholesteatoma relative to skin are structural components.

With respect to the bone proteome of cholesteatomainvolved ossicles, the extensive number of changes 
Table 2 Altered abundances of proteins found in the cholesteatoma matrix proteome relative to healthy ossicles. Positive values indicate increased protein abundance in cholesteatoma. Study ID numbers correlate to Fig. 4

\begin{tabular}{|c|c|c|c|c|}
\hline Protein name (study ID number) & $\begin{array}{l}\text { NCBI gene } \\
\text { ID }\end{array}$ & $\begin{array}{l}\text { Number of unique } \\
\text { peptides }\end{array}$ & $\begin{array}{l}\text { Percent protein } \\
\text { coverage }(\%)\end{array}$ & $\begin{array}{l}\text { Relative protein abundance } \\
\text { Normal ossicles }\end{array}$ \\
\hline Isocitrate dehydrogenase 2 (199) & $\mathrm{IDH} 2$ & 3 & 4.6 & 11.02 \\
\hline Creatine kinase B type (187) & CKB & 3 & 9.7 & 7.81 \\
\hline Thioredoxin domain-co8ntaining protein 5 (290) & TXNDC5 & 3 & 12.0 & 6.76 \\
\hline Cytoskeleton-associated protein 4 (379) & CKAP4 & 2 & 4.8 & 4.64 \\
\hline Protein disulfide-isomerase A6 (166) & PDIA6 & 5 & 15.2 & 4.45 \\
\hline Ribosome binding protein 1 (251) & RRBP1 & 3 & 2.4 & 4.19 \\
\hline Peptidyl-prolyl cis-trans isomerase (368) & FKBP10 & 2 & 4.5 & 4.06 \\
\hline ATP synthase subunit a, mitochondrial (97) & ATP5A1 & 9 & 21.2 & 3.92 \\
\hline Superoxide dismutase, mitochondrial (377) & SOD2 & 2 & 17.2 & 3.92 \\
\hline 60S ribosome L14 (298) & RPL14 & 2 & 10.7 & 3.73 \\
\hline Stress-10 protein, mitochondrial (290) & HSPA9 & 3 & 12.0 & 3.73 \\
\hline Guanine nucleotide binding protein subunit $\beta-2$ (283) & GNB2L1 & 4 & 18.3 & 3.63 \\
\hline Malate dehydrogenase 2 (165) & $\mathrm{MDH} 2$ & 6 & 25.4 & 3.62 \\
\hline Serine protease inhibitor $\mathrm{H} 1$ (248) & SERPINH1 & 4 & 14.6 & 3.50 \\
\hline Dolichyl-diphosphooligosaccharide-protein glycosyltransferase (284) & DDOST & 3 & 7.7 & 3.48 \\
\hline Elongation factor Tu (253) & TUFM & 3 & 10.6 & 3.45 \\
\hline ATP synthase subunit $\beta$, mitochondrial (105) & ATP5B & 6 & 14.6 & 3.41 \\
\hline ADP/ATP translocase 2 (134) & SLC25A5 & 8 & 24.8 & 3.37 \\
\hline Neutrophil elastase (269) & ELANE & 2 & 6.7 & 3.31 \\
\hline Cathepsin G (113) & CTSG & 5 & 22.4 & 3.29 \\
\hline Staphylococcal nuclease domain-containing protein 1 (386) & SND1 & 2 & 2.5 & 3.28 \\
\hline Very long chain specific acyl-CoA dehydrogenase (354) & ACADVL & 2 & 3.5 & 3.26 \\
\hline Coatamer subunit gamma-1 (323) & COPG1 & 2 & 2.6 & 3.13 \\
\hline Prohibitin-2 (358) & PHB2 & 2 & 9.7 & 2.99 \\
\hline Lamin-B1 (396) & LMNB1 & 2 & 3.4 & 2.98 \\
\hline Valine_tRNA ligase (299) & VARS & 2 & 2.1 & 2.97 \\
\hline Aspartate aminotransferase, mitochondrial (344) & GOT2 & 2 & 5.1 & 2.91 \\
\hline 3-Hydroxyacyl-coA dehydrogenase type 2 (267) & HSD17B10 & 2 & 12.3 & 2.89 \\
\hline Membrane primary amine oxidase (225) & $\mathrm{AOC} 3$ & 4 & 3.7 & -3.04 \\
\hline Keratin type II cytoskeletal 8 (337) & KRT8 & 3 & 6.2 & -3.59 \\
\hline Collagen VIII, a-1 chain (208) & COL8A & 3 & 5.0 & -3.66 \\
\hline Cochlin (17) & $\mathrm{COCH}$ & 11 & 20.4 & -4.00 \\
\hline Keratin type II cytoskeletal 7 (164) & KRT7 & 6 & 13.4 & -4.70 \\
\hline Tenascin-X (336) & TNXB & 3 & 0.8 & -4.84 \\
\hline Serum amyloid P-component (93) & APCS & 5 & 21.5 & -5.96 \\
\hline
\end{tabular}

mirrors the complexity of bone as an organ. Many of these changes relate to increased metabolic function, protein degradation, and inflammatory response and less so with structural components. A number of the proteins are constituents of the endoplasmic reticulum, which is abundant in osteoblasts for their synthetic function. Among the proteins with functional ontologic duties, Tenascin-X (TNXB), Serine protease inhibitor $\mathrm{H} 1$
(SERPINH1), and Neutrophil elastase (ELANE), are known factors in bone remodeling. TNXB, decreased in cholesteatoma-involved bone relative to normal ossicles and one of the proteins common to bone and soft tissue analyses, stabilizes extracellular matrix and collagen fibril formation; reduced TNXB is associated with abnormal collagen and elastin deposition and extracellular maturation [32-34]. Whether altered TNXB levels in our study 
reflect a pathologic or reactive change to cholesteatoma remains to be seen. SERPINH1 is a serine protease inhibitor molecular chaperone with critical function in endochondral bone and cartilage formation [35]. Elevated SERPINH1 in cholesteatoma-involved ossicles suggests either increased bone remodeling or repair of the affected bones. This is also likely the scenario for our observation of increased ELANE in cholesteatoma-involved bone. Involved in innate cellular immune processes, ELANE also functions in Collagen VI breakdown and matrix metalloprotease activation [36]. As noted in the soft tissue analysis, proteins present at increased abundances in cholesteatoma-involved bone may also represent markers of residual disease. Cytoskeleton-associated protein 4 (CKAP4) primarily functions in skin development and maintenance [37], implying its presence in cholesteatomainvolved bone correlates with presence of invasive disease.

Limitations of our study center on the use of a largescale proteomic approach to the identification of these altered protein abundances. We found several abundance differences between cholesteatoma matrix and skin compared to the difference between matrix and mucosa. This is challenging to explain but may represent an alteration in protein composition in epidermis as cholesteatoma matures. One possible reason for this is that the local environment in the middle ear is different from that of the exposed post-auricular region. Alternatively, dermal elements could be underrepresented or absent in cholesteatoma while they would not be present in mucosa since there is no appreciable submucosa in the middle ear. Yet another consideration is the inability to measure proteins present in very low abundances, since the peptide fragments from these proteins fail to reach detection thresholds due to the presence of peptides from highly expressed proteins. This remains a challenge to all proteomic studies, although mass spectrometrybased techniques have a wider dynamic range than traditional 2DGE [10, 38].

Additional limitations specific to biomarker discovery studies warrant mention. Identification of disease biomarkers represents a chief objective of proteomics, though to date it as been difficult to carry putative biomarkers through to clinical practice, mainly due to variation between populations and study validity [39]. Discovery phase studies generally use samples containing well-defined and discrete disease states that do not necessarily reflect early, subclinical disease, which would be the ideal target for disease prevention. Similarly, patients with advanced disease often present in a condition that does not match the disease state used to identify the biomarker. Current candidates discovered with large scale proteomic approaches currently under inquiry for roles in clinical diagnostics and disease management include proteins altered in COPD [40], lung cancer [40, 41], gastrointestinal malignancy [42], transplant rejection [43], metabolic diseases [44]. Nonetheless, at present no single protein biomarker discovered through proteomics demonstrates sufficient accuracy to predict disease in a subclinical state [45]. Multiplex approaches to biomarker development present promise to increase understanding of diseases by simultaneously testing multiple proteins and observing abundance changes in concert, and to provide biomarker panels with increased accuracy [45].

\section{Conclusions}

In conclusion, we used a large-scale, two armed proteomic approach to identify and quantify the changes in protein abundance levels in cholesteatoma matrix relative to normal middle ear mucosa and post-auricular skin, as well as in ossicles invaded by cholesteatoma compared to normal ossicles. Given the quantity of proteins involved in epithelial integrity and metabolism, our results provide several avenues for future research into cholesteatoma pathophysiology. Moreover, our inclusion of middle ear mucosa noted novel protein increases in matrix compared to post-auricular skin. One of the exciting findings in our study is the large number of potential biomarkers to use for evaluating residual or recurrent disease, in particular BLMH, TYMP, FLBP5, FLG/FLG2, and CKAP4. Our results describe several accessible proteins that could be used, if future validation demonstrates they are present in altered abundances in cholesteatoma compared to nascent mucosa, as potential therapeutic or diagnostic targets with the aim of reducing recurrent or residual disease and the need for second look tympanomastoidectomy. The greatest challenge with cholesteatoma surgery is eradicating disease without compromising function. Ideally, future cholesteatoma surgery could incorporate intraoperative or postoperative histopathologic margin evaluation using these biomarkers to reduce residual disease and determine timing of second look surgery.

\section{Competing interests}

The authors declare that they have no competing interests.

\section{Authors' contributions}

DRR developed the study protocol, prepared ethics approval, performed molecular analysis, analyzed data, and drafted the manuscript. PSP assisted with study design, data analysis, sample collection, and manuscript development. JKC contributed to study design, ethics application preparation, sample collection, data analysis, and manuscript preparation. All authors read and approved the final manuscript.

\section{Acknowledgments}

The authors thank the University of Calgary Office of Surgical Research (Calgary Surgical Research Development Fund) and the Campbell MacLaurin Foundation for Hearing Research for providing funding to support this study. We also thank Dr. Karl Riabowol for providing laboratory facilities to prepare tissue lysates, Dr. Lorne Clarke for technical advice on bone proteome isolation, and the University of Victoria Genome BC Protein Centre for their expertise in proteomic analysis. 


\section{Funding}

University of Calgary Surgical Research Development Fund, Campbell McLaurin Foundation for Hearing Research.

\section{Received: 3 June 2015 Accepted: 10 November 2015}

Published online: 25 November 2015

\section{References}

1. Prasad SC, Shin SH, Russo A, Di Trapani G, Sanna M. Current trends in the management of the complications of chronic otitis media with cholesteatoma. Curr Opin Otolaryngol Head Neck Surg. 2013;21(5):446-54.

2. Mishiro $Y$, Sakagami M, Kitahara T, Kondoh $K$, Okumura S. The investigation of the recurrence rate of cholesteatoma using Kaplan-Meier survival analysis. Otol Neurotol. 2008;29(6):803-6.

3. Jung JY, Chole RA. Bone resorption in chronic otitis media: the role of the osteoclast. ORL J Otorhinolaryngol Relat Spec. 2002;64:95-107.

4. Olszewska E, Wagner M, Bernal-Sprekelsen M, Ebmeyer J, Dazert S, Hildmann $\mathrm{H}$, et al. Etiopathogenesis of cholesteatoma. Eur Arch Otorhinolaryngol. 2004;261(1):6-24.

5. Morales SD, Penido N de O, da Silva NID, Stavale JN, Guilherme A, Fukuda Y. Matrix metalloproteinase 2: an important genetic marker for cholesteatomas. Braz J Otorhinolaryngol. 2007;73:51-7.

6. Nason R, Jung JY, Chole RA. Lipopolysaccharide-induced osteoclastogenesis from mononuclear precursors: a mechanism for osteolysis in chronic otitis. J Assoc Res Otolaryngol. 2009;10:151-60

7. Louw L. Acquired cholesteatoma pathogenesis: stepwise explanations. J Laryngol Otol. 2010;124(6):587-93.

8. Kuo CL. Etiopathogenesis of acquired cholesteatoma: prominent theories and recent advances in biomolecular research. Laryngoscope. 2015;125(1):234-40.

9. Aggarwal K, Choe LH, Lee KH. Shotgun proteomics using the iTRAQ isobaric tags. Brief Funct Genomic Proteomic. 2006;5(2):112-20.

10. Feist $P$, Hummon AB. Proteomic challenges: sample preparation techniques for microgram-quantity protein analysis from biological samples. Int J Mol Sci. 2015:16(2):3537-63.

11. Kim JL, Jung HH. Proteomic analysis of cholesteatoma. Acta Otolaryngol. 2004:124(7):783-8

12. Lee Y, Jang S, Min JK, Lee K, Sohn KC, Lim JS, et al. S100A8 and S100A9 are messengers in the crosstalk between epidermis and dermis modulating a psoriatic milieu in human skin. Biochem Biophys Res Commun. 2012;423(4):647-53.

13. Hattori F, Kiatsurayanon C, Okumura K, Ogawa H, Ikeda S, Okamoto K, et al. The antimicrobial protein S100A7/psoriasin enhances the expression of keratinocyte differentiation markers and strengthens the skin's tight junction barrier Br J Dermatol. 2014:171(4):742-53.

14. Abtin A, Eckhart L, Mildner M, Gruber F, Schröder JM, Tschachler E. Flagellin is the principal inducer of the antimicrobial peptide S100A7c (psoriasin) in human epidermal keratinocytes exposed to Escherichia coli. FASEB J. 2008;22(7):2168-76.

15. Suminami $Y$, Kishi F, Sekiguchi $K$, Kato H. Squamous cell carcinoma antigen is a new member of the serine protease inhibitors. Biochem Biophys Res Commun. 1991;181(1):51-8.

16. Vidalino L, Doria A, Quarta S, Zen M, Gatta A, Pontisso P. SERPINB3, apoptosis and autoimmunity. Autoimmun Rev. 2009;9(2):108-12.

17. Quarta S, Vidalino L, Turato C, Ruvoletto M, Calabrese F, Valente M, et al. SERPINB3 induces epithelial-mesenchymal transition. J Pathol. 2010;221(3):343-56.

18. Ho KY, Huang HH, Hung KF, Chen JC, Chai CY, Chen WT, et al. Cholesteatoma growth and proliferation: relevance with serpin B3. Laryngoscope. 2012;122(12):2818-23.

19. Schwartz DR, Homanics GE, Hoyt DG, Klein E, Abernethy J, Lazo JS. The neutral cysteine protease bleomycin hydrolase is essential for epidermal integrity and bleomycin resistance. Proc Natl Acad Sci USA. 1999;96(8):4680-5.

20. Stammberger M, Bujía J, Kastenbauer E. Alteration of epidermal differentiation in middle ear cholesteatoma. Am J Otol. 1995;16(4):527-31.

21. Min HJ, Park CW, Jeong JH, Cho SH, Kim KR, SH L. Comparative analysis of the expression of involucrin, filaggrin and cytokeratin 4, 10, 16 in cholesteatoma. Korean J Audiol. 2012;16(3):124-9.

22. Makino T, Mizawa M, Yamakoshi T, Takaishi M, Shimizu T. Expression of filaggrin-2 protein in the epidermis of human skin diseases: a comparative analysis with filaggrin. Biochem Biophys Res Commun. 2014;449(1):100-6.

23. Huisman MA, De Heer E, Grote JJ. Survival signaling and terminal differentiation in cholesteatoma epithelium. Acta Otolaryngol. 2007;127(4):424-9.
24. Ikeda R, Tajitsu Y, Iwashita K, Che XF, Yoshida K, Ushiyama M, et al Thymidine phosphorylase inhibits the expression of proapoptotic protein BNIP3. Biochem Biophys Res Commun. 2008;370(2):220-4

25. Louw L. Acquired cholesteatoma: summary of the cascade of molecular events. J Laryngol Otol. 2013;127(6):542-9.

26. Dallaglio K, Marconi A, Truzzi F, Lotti R, Palazzo E, Petrachi T, et al. E-FABP induces differentiation in normal human keratinocytes and modulates the differentiation process in psoriatic keratinocytes in vitro. Exp Dermatol. 2013;22(4):255-61.

27. Hagens G, Roulin K, Hotz R, Saurat JH, Hellman U, Siegenthaler G. Probable interaction between S100A7 and E-FABP in the cytosol of human keratinocytes from psoriatic scales. Mol Cell Biochem. 1999;192(1-2):123-8.

28. Ruse M, Broome AM, Eckert RL. S100A7 (psoriasin) interacts with epidermal fatty acid binding protein and localizes in focal adhesion-like structures in cultured keratinocytes. J Invest Dermatol. 2003;121(1):132-41.

29. Kusakari Y, Ogawa E, Owada Y, Kitanaka N, Watanabe H, Kimura M, et al. Decreased keratinocyte motility in skin wound on mice lacking the epiderma fatty acid binding protein gene. Mol Cell Biochem. 2006;284(1-2):183-8.

30. Hartlieb E, Kempf B, Partilla M, Vigh B, Spindler V, Waschke J. Desmoglein 2 is less important than desmoglein 3 for keratinocyte cohesion. PLoS One. 2013;8(1), e53739.

31. Koizumi H, Suzuki H, Ohbuchi T, Kitamura T, Hashida K, Nakamura M. Increased permeability of the epithelium of middle ear cholesteatoma. Clin Otolaryngol. 2015;40(2):106-14.

32. Zweers MC, van Vlijmen-Willems IM, van Kuppevelt TH, Mecham RP, Steijlen PM, Bristow J, et al. Deficiency of tenascin-X causes abnormalities in dermal elastic fiber morphology. J Invest Dermatol. 2004:122(4):885-91.

33. Egging D, van den Berkmortel F, Taylor G, Bristow J, Schalkwijk J. Interactions of human tenascin-X domains with dermal extracellular matrix molecules. Arch Dermatol Res. 2007;298(8):389-96.

34. Egging D, van Vlijmen-Willems I, van Tongeren T, Schalkwijk J, Peeters A Wound healing in tenascin- $X$ deficient mice suggests that tenascin- $X$ is involved in matrix maturation rather than matrix deposition. Connect Tissue Res. 2007;48(2):93-8.

35. Masago Y, Hosoya A, Kawasaki K, Kawano S, Nasu A, Toguchida J, et al. The molecular chaperone Hsp47 is essential for cartilage and endochondral bone formation. J Cell Sci. 2012;125(Pt 5):1118-28.

36. Kielty CM, Lees M, Shuttleworth CA, Woolley D. Catabolism of intact type VI collagen microfibrils: susceptibility to degradation by serine proteinases. Biochem Biophys Res Commun. 1993:191(3):1230-6.

37. Koster Ml. p63 in skin development and ectodermal dysplasias. J Invest Dermatol. 2010;130(10):2352-8

38. Choe LH, Aggarwal K, Franck Z, Lee KH. A comparison of the consistency of proteome quantitation using two-dimensional electrophoresis and shotgun isobaric tagging in Escherichia coli cells. Electrophoresis. 2005;26(12):2437-49.

39. Lin JL, Bonnichsen MH, Nogeh EU, Raftery MJ, Thomas PS. Proteomics in detection and monitoring of asthma and smoking-related lung diseases. Expert Rev Proteomics. 2010;7(3):361-72

40. Ohlmeier S, Vuolanto M, Toljamo T, Vuopala K, Salmenkivi K, Myllärniemi M, et al. Proteomics of human lung tissue identifies surfactant protein $A$ as a marker of chronic obstructive pulmonary disease. J Proteome Res. 2008;7(12):5125-32.

41. Bajtarevic A, Ager C, Pienz M, Klieber M, Schwarz K, Ligor M, et al. Noninvasive detection of lung cancer by analysis of exhaled breath. BMC Cancer. 2009:9:348

42. Kuramitsu Y, Nakamura K. Proteomic analysis of cancer tissues: shedding light on carcinogenesis and possible biomarkers. Proteomics. 2006:6(20):5650-61.

43. Cohen Freue GV, Meredith A, Smith D, Bergman A, Sasaki M, Lam KK, et al. Biomarkers in Transplantation and the NCE CECR Prevention of Organ Failure Centre of Excellence Teams. Computational biomarker pipeline from discovery to clinical implementation: plasma proteomic biomarkers for cardiac transplantation. PLoS Comput Biol. 2013;9(4), e1002963.

44. Randall DR, Sinclair GB, Colobong KE, Hetty E, Clarke LA. Heparin cofactor Il-thrombin complex in MPS I: a biomarker of MPS disease. Mol Genet Metab. 2006:88(3):235-43.

45. Schiess R, Wollscheid B, Aebersold R. Targeted proteomic strategy for clinical biomarker discovery. Mol Oncol. 2009;3(1):33-44. 